\title{
NIVEL DE INGRESOS Y USO DE TARJETAS DE CRÉDITO EN PERÚ
}

\author{
LEVEL OF INCOME AND USE OF CREDIT CARDS IN PERU
}

\section{RESUMEN}

Objetivo: Determinar si el nivel de ingreso explica el uso de tarjetas de crédito en el Perú. Método: Se utilizó un modelo Logit para estimar la probabilidad de uso de tarjetas de crédito en aquellos individuos que cuentan con una tarjeta. Resultados: Los ingresos tienen un efecto positivo y estadísticamente significativo en el uso de tarjetas de crédito; sin embargo, en algunos rubros, como en el gasto de alimentos, no se observan diferencias significativas en el uso de la tarjeta de crédito con relación a los ingresos laborales mensuales. Conclusión: La población con menores ingresos tiende a usar la tarjeta de crédito en igual proporción que los segmentos con mayores ingresos para determinados gastos.

Palabras clave: Acceso, endeudamiento, ingresos, segmentos, tarjeta de crédito.
VLADIMIR RodRÍGUez CAIRO Universidad Nacional Mayor de San Marcos Lima, Perú

ORCID: https://orcid.org/0000-0001-9971-0405 Correo electrónico: vrodriguezc@unmsm.edu.pe

Raphael Ángel Saldaña Pacheco Ministerio de Economía y Finanzas Lima, Perú ORCID: https://orcid.org/0000-0002-2016-3486 Correo electrónico: raphael.saldana@pucp.edu.pe

Johanna Marissa Yancari Cueva Instituto de Estudios Peruanos Lima, Perú

ORCID: https://orcid.org/0000-0001-9330-5978 Correo electrónico: jyancari@iep.org.pe

[Recibido: 30/09/2020 Aceptado: 04/12/2020 Publicado: 28/12/2020]

\begin{abstract}
Objective: Determine if the income level explains the use of credit cards in Peru. Method: A Logit model was used to estimate the probability of use credit cards on those persons who have a card. Results: The Income has a positive effect and statistically significant on credit card use, however, in some areas, as the food spend, it doesn't observe significant differences in the credit card use related to monthly work income. Conclusions: The population with lower income tends to use the credit card in equal proportion as the higher income segments for certain expenses.
\end{abstract}

Keywords: Access; indebtedness; income; segments; credit card. 


\section{INTRODUCCIÓN}

El crédito es una operación financiera a través de la cual las entidades financieras ponen a disposición del cliente una determinada cantidad de fondos, bajo ciertas condiciones, durante un plazo; y los intereses se cargan sobre las cantidades realmente dispuestas o utilizadas. De acuerdo con el Diccionario panhispánico del español jurídico, una de las acepciones del vocablo crédito es la siguiente: "Cantidad de dinero, o cosa equivalente, que alguien debe a una persona o entidad, y que el acreedor tiene derecho de exigir y cobrar" (Real Academia Española, 2020, lema 2, definición 1).

De manera que el crédito es una operación que permite a las personas acceder a diversos bienes de consumo, vestimenta o vivienda, entre otros; por ello, los tipos de créditos son: de consumo, hipotecarios, etc. Sin embargo, el uso de estos instrumentos financieros conlleva costos y riesgos que deben ser considerados por el cliente. Por ello, al momento de tomar decisiones financieras al respecto, hay una probabilidad de cometer errores y de enfrentar contingencias inesperadas.

Una de las formas para la obtención del crédito de consumo son las tarjetas de crédito, instrumento financiero que ha impulsado la expansión del crédito de consumo e, incluso, el sobreendeudamiento de la población. En el Diccionario panhispánico del español jurídico se define a la tarjeta de crédito como el "documento de sustancia plástica que lleva incorporada una banda magnética que sirve para identificar electrónicamente a su titular y la cuenta bancaria a la que la tarjeta se asocia, y que proporciona un crédito a su titular para satisfacer obligaciones dinerarias" (Real Academia Española, 2020).

En ese sentido, las tarjetas de crédito permiten a su titular efectuar diversas operaciones con cargo a una línea de crédito revolvente. Evidentemente, el usuario puede efectuar pagos en establecimientos afiliados al momento de adquirir bienes o servicios, pagar obligaciones, hacer uso del servicio de disposición de efectivo u otros servicios asociados, dentro de los límites y condiciones estipulados en el contrato celebrado entre la empresa emisora y el titular; este último está obligado a devolver o pagar el dinero o importe de los productos adquiridos, así como otros cargos, conforme a lo establecido en el respectivo contrato.

Así, en el Perú, tal y como señalan Sánchez y Cisneros (2020), vemos que el mercado de tarjetas de crédito se ha desarrollado en gran medida permitiendo el acceso de más personas al sistema financiero. Asimismo, esta situación fue posible tanto por los avances tecnológicos llevados a cabo por las empresas del sistema financiero que conllevaron a mejoras en los procesos de selección y medición del riesgo de nuevos clientes, como a las características de la población producto de un entorno económico y laboral favorable.

En particular observamos que la experiencia internacional señala que las características de la población son relevantes para entender el uso de tarjetas de crédito. Así, Zandi et al. (2019) encuentran evidencia sobre la relación que existe entre las características de la población con el acceso y uso de tarjetas de crédito en Malasia, hallando que el nivel de ingresos de los individuos afecta el uso de tarjetas de crédito, incluso más que la edad, el género y el nivel educativo. Además, Chan (1997) halla que las características demográficas importan para explicar el uso de la tarjeta de crédito y encuentra que el factor explicativo más relevante es el nivel de ingreso del individuo. En caso similar, Kim y DeVaney (2001) estudian la relación entre el nivel de ingresos del titular de la tarjeta de crédito y el monto adeudado en tarjeta de crédito; encontraron que los consumidores con altos ingresos tienen un mayor límite de crédito en sus tarjetas, lo que guarda relación con su poder adquisitivo y, así, mantienen grandes saldos pendientes de pago.

Arango y Cardona-Sosa (2019), al investigar los determinantes del uso de la tarjeta Crédito Fácil Codensa (Colombia), concluyeron que los ingresos del titular de la tarjeta son un aspecto decisivo de los montos adquiridos a través de esta. Caso parecido es el hallado por Bertaut y Haliassos (2006), quienes coligieron que el nivel de ingresos influye en la tenencia de una tarjeta de crédito; sin embargo, a diferencia de otras investigaciones, determinaron que el nivel de estudios del cliente financiero afecta la probabilidad de la posesión de tarjetas de crédito, ya que está íntimamente relacionado con el nivel de ingresos del individuo.

Desde tal perspectiva, este artículo se enfoca en la implicancia del nivel de ingresos del titular de la tarjeta de crédito y su uso en el Perú. De manera que, se formula la siguiente interrogante de investigación: ¿Los ingresos de la población explican el uso de tarjetas de crédito en el Perú? La hipótesis que responde a la pregunta es: Los ingresos de la población explican el uso de tarjetas de crédito en Perú.

\section{MATERIALES Y MÉTODOS}

El enfoque del estudio fue cuantitativo con análisis cualitativo y el alcance fue descriptivo, relacional y explicativo. Descriptivo, pues se describieron las características relacionadas con el acceso a productos en el sistema financiero y con el nivel de ingresos laborales de los usuarios de manera general; y, específicamente, se estudiaron las características de los usuarios de tarjetas de crédito tomando en consideración su nivel de ingresos. Relacional, debido a que se asociaron las características de la población, especialmente el nivel de ingreso laboral mensual (controlando por género 
y edad), con el uso de las tarjetas de crédito. Explicativo, porque se estableció la implicancia del ingreso en el uso de tarjetas de crédito.

De esta manera, la población objetivo es la conformada por personas mayores de 18 años que tienen tarjetas de crédito a nivel nacional; y se tiene como unidad de análisis a los individuos. Los datos fueron obtenidos a partir de la Encuesta Nacional de Hogares (ENAHO) del año 2019, recolectada de manera anual por el Instituto Nacional de Estadística e Informática, que permite obtener estimaciones de las características sociodemográficas de la población en el Perú a nivel nacional. La ENAHO cuenta con un diseño muestral probabilístico y multietápico que, para el año 2019, contempla una muestra de 36994 viviendas y 82 672 individuos con al menos 18 años de edad.

Con los datos de la ENAHO 2019, se procedió tanto al análisis descriptivo como al análisis estadístico inferencial. En el análisis descriptivo, se examinó la composición de la población adulta en función al acceso a productos financieros y a la tenencia de una tarjeta de crédito, así como su uso, tomando en cuenta el nivel de ingresos laborales mensuales del individuo, con el propósito de observar si existe algún comportamiento asociado a las características de la población, con énfasis en el nivel de ingresos del individuo.

Luego, se estimó un modelo Logit para determinar la probabilidad de que aquellos individuos que tienen una tarjeta de crédito la utilizan. Esta variable dependiente se denota como $\mathrm{Y}_{\mathrm{i}}$, la cual tiene carácter dicotómico debido a la naturaleza mutuamente excluyente que supone el evento referido al uso de tarjeta de crédito efectuado por el i-ésimo individuo con una probabilidad "p". De esta manera la variable $Y_{i}$ se comporta de la siguiente forma:

$\mathrm{Y}_{\mathrm{i}}=\left\{\begin{array}{lll}1 & \text { si el individuo " } \mathrm{i} " \text { usa tarjeta de crédito, } & \text { con probabilidad } \mathrm{p} \\ 0 & \text { en caso contrario, } & \text { con probabilidad } 1-\mathrm{p}\end{array}\right.$

De esta manera, el modelo Logit a estimar se detalla así:

$$
\operatorname{Logit}\left[\operatorname{Pr}\left(Y_{i}=1 \mid X_{i}\right)\right]=\ln \left(\frac{\operatorname{Pr}\left(Y_{i}=1\right)}{1-\operatorname{Pr}\left(Y_{i}=1\right)}\right)=X_{i}^{\prime} \beta
$$

En donde, el vector de características $\mathrm{X}_{\mathrm{i}}$ está compuesto por el nivel de ingresos laborales mensuales $y$, a manera de controles, se utilizaron las variables edad y el género. Posteriormente, se predijeron las probabilidades de uso de tarjeta de crédito de los individuos que poseen una, considerando las diferencias por ingreso laboral mensual.

\section{RESULTADOS}

En la tabla 1, se aprecia que, hasta febrero de 2019, en el Perú existían 7988134 tarjetas de crédito de bancos y financieras por créditos de consumo, de las cuales, el $76 \%$ correspondía a bancos y el $24 \%$, a financieras. De los 11

Tabla 1

Perú: Tarjetas de crédito por entidad financiera (febrero, 2019)

\begin{tabular}{lcc}
\hline \multicolumn{1}{c}{ EMPRESA } & N. ${ }^{\circ}$ de Titulares & Participación (\%) \\
\hline B. BBVA Perú & 464467 & $7,6 \%$ \\
B. de Comercio & 1159 & $0,0 \%$ \\
B. de Crédito del Perú (con sucursales en exterior) & 973714 & $16,0 \%$ \\
B. Pichincha & 286733 & $4,7 \%$ \\
B. Interamericano de Finanzas & 98847 & $1,6 \%$ \\
Scotiabank Perú & 498426 & $8,2 \%$ \\
Interbank (con sucursales en el exterior) & 977719 & $16,1 \%$ \\
B. GNB & 6055 & $0,1 \%$ \\
B. Falabella Perú & 1526668 & $25,1 \%$ \\
B. Ripley & 1197757 & $19,7 \%$ \\
B. Azteca Perú & 40285 & $0,7 \%$ \\
\hline Total: Empresas bancarias & 6071830 & $100,0 \%$ \\
\hline CrediScotia Financiera & 778012 & $40,6 \%$ \\
Financiera Oh! & 1138292 & $59,4 \%$ \\
\hline Total: Empresas financieras & 1916304 & $100,0 \%$ \\
\hline TOTAL: BANCOS + FINANCIERAS & 7988134 & \\
\hline
\end{tabular}

Fuente: Elaboración propia a partir de los datos de la Superintendencia de Banca, Seguros y Administradoras Privadas de Fondos de Pensiones (SBS). 
bancos que operaban en el país, solo cinco (Falabella, Crédito, Interbank, Ripley y Scotiabank) concentraban el $85,2 \%$ del mercado de tarjetas de crédito. Sin embargo, esta tabla no toma en cuenta si una persona tiene más de una tarjeta de crédito.

No obstante, sobre la base de los datos de la ENAHO 2019, se observa que el acceso a productos en el sistema financiero por parte la población adulta (mayor a 18 años) es de $42,3 \%$, y que la proporción de acceso se incrementa conforme aumentan los ingresos del individuo. Entonces, mientras menos de la tercera parte de los individuos que cuentan con ingresos laborales mensuales menores a S/ 1000 tienen acceso a productos en el sistema financiero, casi la totalidad de la población que alcanza niveles de ingreso superiores a S/ 8000 (94\%) accede a estos servicios, lo cual muestra que existe heterogeneidad en el nivel de acceso a productos en el sistema financiero por parte de la población (tabla 2).

En la tabla 3, se observa que solo el 17,8\% de los adultos (1,6 millones) que acceden a productos en el sistema financiero tiene tarjetas de crédito. Además, considerando el número de tarjetas de crédito en el sistema financiero y el número de personas con tarjeta de crédito, el adulto que accede a tarjetas de crédito tendría en promedio cinco de estas. Asimismo, se aprecia una tendencia similar en el comportamiento del acceso al sistema financiero y el nivel de ingresos de la población. Así, la proporción de tenencia de tarjeta de crédito de las personas que obtienen ingresos laborales mensuales menores a S/ 1000 es casi 7 veces menor a lo observado en el grupo de personas con ingresos mayores a S/ 8000. Sin embargo, solo 7 de cada 10 personas en el nivel de mayores ingresos laborales (más de S/ 8000) cuenta con una tarjeta de crédito, lo que muestra que existen brechas de atención, incluso, en este nivel de ingresos.

Con relación al uso de tarjetas de crédito, se determinó que el $50 \%$ de aquellos que tienen tarjeta de crédito la usaron durante el año 2019 (tabla 4). Se aprecia que la proporción de uso con relación a los ingresos es menor en este grupo. Así, mientras que 4 de cada 10 de los que tienen una tarjeta de crédito en el segmento de menores ingresos

Tabla 2

Acceso de la población a productos en el sistema financiero

- Por nivel de ingreso laboral mensual, mayores de 18 años -

\begin{tabular}{llcc}
\hline & \multicolumn{3}{c}{ Acceso a servicios financieros } \\
\hline \multicolumn{1}{c}{ Nivel de Ingresos } & \multicolumn{1}{c}{ Tiene } & No tiene & $\%$ Tiene \\
\hline Menos de S/ 1000 & 4216008 & 9787127 & $30,1 \%$ \\
Entre S/ 1000 y S/ 2000 & 3878477 & 2852679 & $57,6 \%$ \\
Entre S/ 2000 y S/ 4000 & 870285 & 137830 & $86,3 \%$ \\
Entre S/ 4000 y S/ 8000 & 291573 & 31736 & $90,2 \%$ \\
Más de S/ 8000 & 135938 & 8284 & $94,3 \%$ \\
\hline
\end{tabular}

Fuente: Elaboración propia a partir de datos de la ENAHO (2019)

Tabla 3

Acceso de la población a tarjetas de crédito

- Por nivel de ingreso laboral mensual para los que acceden al sistema financiero -

\begin{tabular}{|c|c|c|c|}
\hline \multicolumn{4}{|c|}{ Tarjeta de Crédito } \\
\hline Nivel de Ingresos & Tiene & No tiene & $\%$ Tenencia \\
\hline Menos de S/ 1000 & 454574 & 3761434 & $10,8 \%$ \\
\hline Entre S/ 1000 y S/ 2000 & 663934 & 3214543 & $17,1 \%$ \\
\hline Entre S/ 2000 y S/ 4000 & 310051 & 560233 & $35,6 \%$ \\
\hline Entre S/ 4000 y S/ 8000 & 148903 & 142670 & $51,1 \%$ \\
\hline Más de S/ 8000 & 92634 & 43304 & $68,1 \%$ \\
\hline & 1670096 & 7722184 & $17,8 \%$ \\
\hline
\end{tabular}

Fuente: Adaptado de Encuesta Nacional de Hogares 2019, Instituto Nacional de Estadística e Informática, 2019 (https://www. inei.gob.pe/) 
la usa; 7 de cada 10 hace lo propio en el segmento de mayores ingresos. Esto muestra diferencias con relación a lo encontrado en el nivel de acceso.

A partir de la ENAHO (2019), se puede reconocer rubros para los cuales el individuo utiliza como medio de pago la tarjeta de crédito. De esta manera, en la tabla 5, se denomina alimentos al gasto de alimentos fuera y dentro del hogar; gastos del hogar, al gasto en lavandería, vestimenta y aseo; mientras que servicios básicos se refiere al gasto en electricidad, agua, teléfono y combustible para cocinar; finalmente, muebles y electrodomésticos trata sobre el gasto en muebles, enseres y artefactos electrodomésticos. Por un lado, los resultados arrojaron que los usos más importantes de la tarjeta de crédito corresponden al rubro de alimentos y gastos del hogar, y en estos casos no se observa diferencias en esta proporción con relación al nivel de ingresos de la población. Por otro lado, el uso de la tarjeta de crédito por parte de los segmentos de ingresos más altos en el pago de servicios básicos y la compra de muebles y electrodomésticos es de mayor importancia, aunque por debajo del $40 \%$.
Respecto al uso de tarjetas de crédito por edad, en la figura 1 , se observa que los más jóvenes quienes usan en menor proporción la tarjeta de crédito. Ello debido a la relación - analizada anteriormente- entre el nivel de ingreso y el uso.

Con respecto al uso de las tarjetas de crédito según el género de las personas, en la figura 2, se observa que las mujeres que tienen tarjeta de crédito la emplean más que los hombres.

De este modo, la estimación del modelo Logit (tabla 6) muestra que hay una relación positiva entre el uso de tarjetas de crédito y el nivel de ingreso laboral mensual.

En la tabla 7 se observa que la probabilidad predicha del uso de tarjetas de crédito guarda una relación positiva con el ingreso laboral mensual. De modo que, cuando el individuo obtiene ingresos laborales mensuales de S/ 500 la probabilidad de uso es de $43 \%$; pero cuando el individuo obtiene ingresos por S/ 8000 , es de $68 \%$.

Tabla 4

Uso de tarjetas de crédito

- Por nivel de ingreso laboral mensual para los que tienen tarjeta de crédito -

\begin{tabular}{lccc}
\hline \multicolumn{1}{c}{ Nivel de Ingresos } & Usa & No usa & $\%$ Usa \\
\hline Menos de S/ 1000 & 188428 & 266146 & $41,5 \%$ \\
Entre S/ 1000 y S/ 2000 & 322623 & 341311 & $48,6 \%$ \\
Entre S/ 2000 y S/ 4000 & 176255 & 133797 & $56,8 \%$ \\
Entre S/ 4000 y S/ 8000 & 91219 & 57684 & $61,3 \%$ \\
Más de S/ 8000 & 64408 & 28226 & $69.5 \%$ \\
\hline
\end{tabular}

Fuente: Adaptado de Encuesta Nacional de Hogares 2019, Instituto Nacional de Estadística e Informática, 2019 (https://www. inei.gob.pe/)

Tabla 5

Uso de tarjetas de crédito

- Por nivel de ingreso laboral mensual según rubros de gasto -

\begin{tabular}{lllll}
\hline & \multicolumn{3}{c}{ Usos de tarjeta de crédito } \\
\hline \multicolumn{1}{c}{ Nivel de Ingresos } & Alimentos & Gastos del hogar & Servicios básicos & Muebles y electrodomésticos \\
\hline Menos de S/ 1 000 & $68 \%$ & $86 \%$ & $15 \%$ & $18 \%$ \\
Entre S/ 1000 y S/ 2000 & $63 \%$ & $83 \%$ & $14 \%$ & $26 \%$ \\
Entre S/ 2000 y S/ 4000 & $69 \%$ & $87 \%$ & $17 \%$ & $32 \%$ \\
Entre S/ 4000 y S/ 8000 & $74 \%$ & $91 \%$ & $22 \%$ & $36 \%$ \\
Más de S/ 8000 & $83 \%$ & $91 \%$ & $33 \%$ & $38 \%$ \\
\hline
\end{tabular}

Fuente: Adaptado de Encuesta Nacional de Hogares 2019, Instituto Nacional de Estadística e Informática, 2019 (https://www.inei.gob.pe/) 


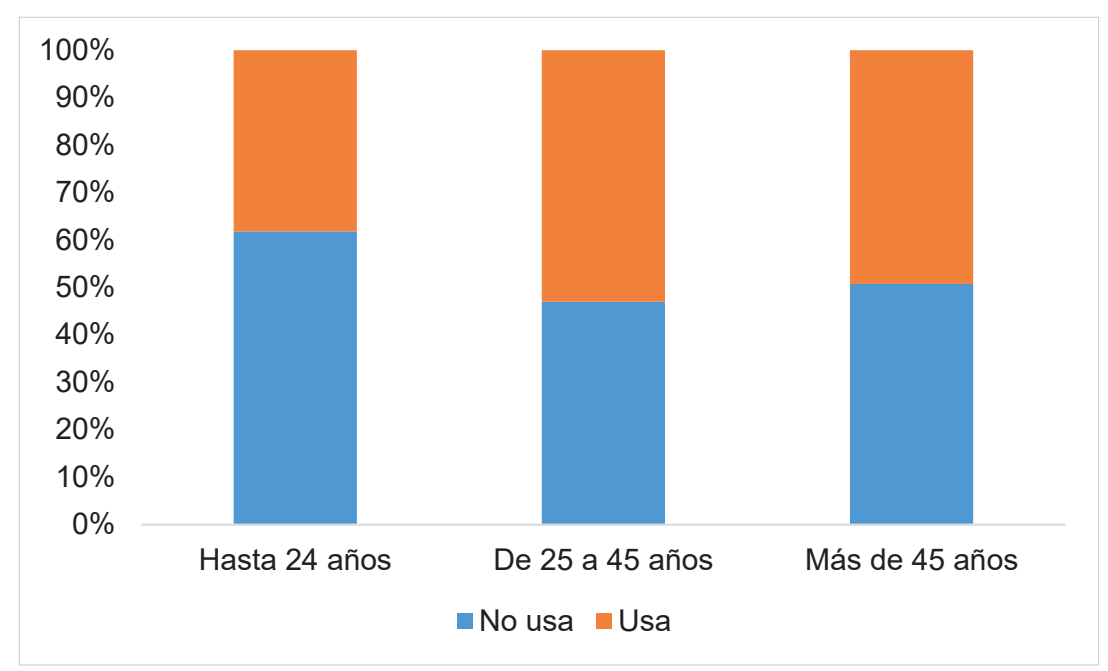

Figura 1. Uso de tarjetas de crédito por edad

Fuente: Adaptado de Encuesta Nacional de Hogares 2019, Instituto Nacional de Estadística e Informática, 2019 (https://www.inei.gob.pe/)

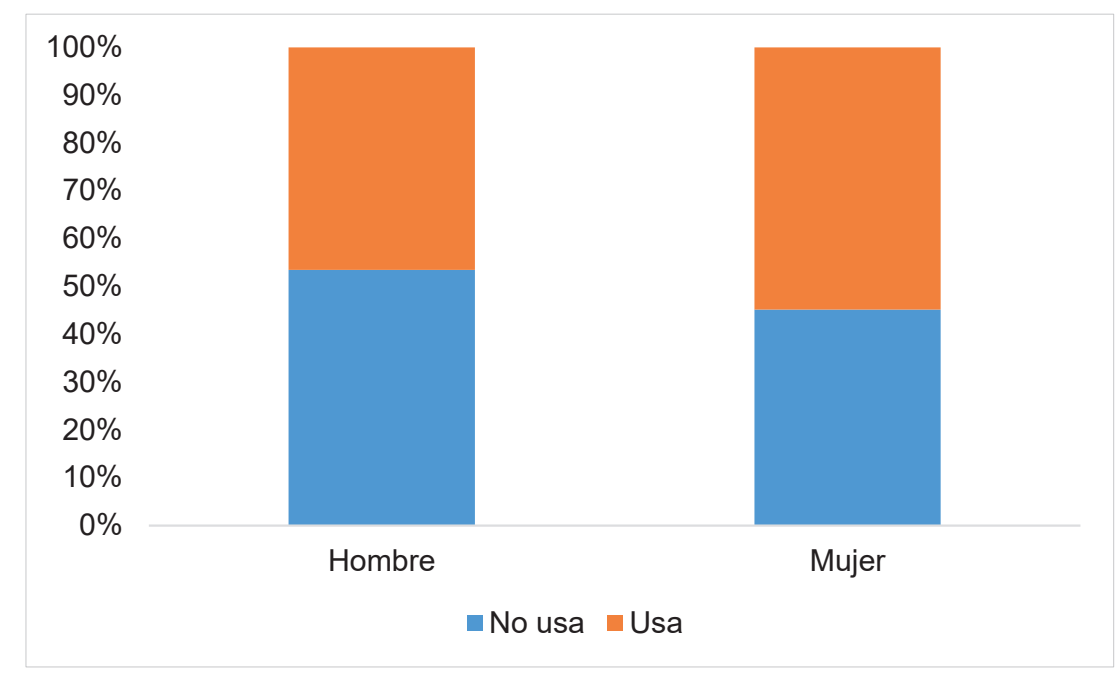

Figura 2. Uso de tarjetas de crédito por género

Fuente: Adaptado de Encuesta Nacional de Hogares 2019, Instituto Nacional de Estadística e Informática, 2019 (https://www.inei.gob.pe/)

\section{DISCUSIÓN}

El bajo nivel de educación financiera existente entre los usuarios es una situación de suma importancia en el Perú. Según la Encuesta Nacional de Demanda de Servicios Financieros y Nivel de Cultura Financiera en el Perú (2016), el conocimiento sobre conceptos financieros básicos, como la tasa de interés, es limitado; solo el 19\% de la población a nivel nacional pudo resolver de manera correcta el cálculo presentado. En las poblaciones más pobres este porcentaje disminuye a $7 \%$. Esto incide directamente en el comportamiento asociado al uso de tarjetas de crédito, siendo así más riesgoso en poblaciones de bajos ingresos con menor educación financiera. Con relación al nivel de educación financiera, Barboza et al. (2016) encontraron que existen diferencias cognitivas en torno al nivel de educación financiera alcanzado por el individuo, basado en género y desempeño educativo. De esta manera, personas con menor desempeño educativo alcanzan menor nivel de educación financiera; y en relación con el género, las mujeres tienen, en promedio, menor nivel de educación financiera independientemente del desempeño educativo obtenido. Además, Annabi et al. (2018) determinaron que, dado que existe bajo nivel de educación financiera entre 
Tabla 6

Resultados estimación Logit (endógena: uso de tarjeta de crédito)

\begin{tabular}{llll}
\hline & \multicolumn{1}{c}{ Valor } & Significancia \\
\hline Ingreso Laboral mensual (S/) & 0,0001371 & $* * *$ & \\
Mujer & 0,4621629 & $* * *$ & \\
Edad & $-0,004075$ & $* * *$ & \\
Constante & $-0,400595$ & \\
\hline Observaciones & 4915 & \\
Población & 1670096 & \\
Grados de libertad & 2114 & \\
F( 3, 2160) & 29,41 & \\
Prob > F & 0 & \\
\hline
\end{tabular}

Fuente: Adaptado de Encuesta Nacional de Hogares 2019, Instituto Nacional de Estadística e Informática, 2019 (https://www.inei.gob.pe/)

Tabla 7

Probabilidad de uso de tarjeta de crédito (por nivel de ingreso)

\begin{tabular}{lcccccc}
\hline & Probabilidad & Error Estándar & $\mathbf{Z}$ & \multicolumn{1}{c}{$\mathbf{P}>|\mathbf{z}|$} & \multicolumn{2}{c}{ Intervalo de confianza (95\%) } \\
\hline S/ 500 & 0,43 & 0,02 & 26,88 & 0 & 0,40 & 0,46 \\
S/ 1000 & 0,45 & 0,02 & 29,55 & 0 & 0,42 & 0,48 \\
S/ 2000 & 0,48 & 0,01 & 34,47 & 0 & 0,45 & 0,51 \\
S/ 4000 & 0,55 & 0,01 & 37,28 & 0 & 0,52 & 0,58 \\
S/ 8000 & 0,68 & 0,02 & 29,65 & 0 & 0,63 & 0,72 \\
\hline
\end{tabular}

Fuente: Adaptado de Encuesta Nacional de Hogares 2019, Instituto Nacional de Estadística e Informática, 2019 (https://www.inei.gob.pe/)

los jóvenes, y estos están expuestos a decisiones de gasto a través de servicios financieros como tarjetas de crédito, créditos o planes de retiro, es necesario contar con más programas de educación financiera para prevenir que tomen malas decisiones al adquirir alguno de estos productos. En este sentido, existen grupos más vulnerables al uso inadecuado de servicios financieros, como las tarjetas de crédito, entre los que se encuentran los jóvenes, las mujeres y la población con mejor nivel educativo, por lo que se debe poner especial interés en la protección de consumidores con estas características. El mejor ecosistema brindado para promover el uso responsable de servicios financieros como las tarjetas de crédito, no solo abarca los conocimientos y capacidades de la demanda, sino que comprende también a la oferta. Así, existen incentivos perversos en el actuar de las empresas de servicios financieros respecto de la presentación de información de sus productos, por lo que es necesario fortalecer la regulación. En esas líneas, Sarin (2019) señala que los bancos tienden a oscurecer el costo real de los productos financieros, por lo que la regulación financiera debe restringir estas prácticas. Asimismo, indica que siempre existe una propensión a que los bancos traten de evitar mayor regulación bajo el pretexto que esto afectará el costo de los servicios financieros y será trasladado, finalmente, al consumidor. Por ello, la regulación financiera debe considerar, en el diseño de políticas públicas, estos comportamientos por parte de la oferta de servicios financieros y actuar en favor del bienestar del consumidor. Esta investigación determinó que la proporción de uso de las tarjetas de crédito por parte de la población con menores ingresos laborales es de $41 \%$; mientras que para la población con ingresos más altos es alrededor del $70 \%$. Esto no implicaría un problema si no fuera porque el segmento de bajos ingresos se refiere a la población cuyos ingresos son menores a S/ 1000, y el de altos ingresos percibe más de $\mathrm{S} / 8000$. Esta gran diferencia en ingresos y no en proporción de uso, aunado al bajo nivel de educación financiera en poblaciones más pobres, incrementa la escala del problema potencial en torno al uso responsable de los productos financieros como la tarjeta de crédito. En la futura agenda de investigación sobre el tema abordado debe orientarse a la realización de estudios que ahonden en el comportamiento deudor al usar las tarjetas de crédito de tal forma que, a partir de dicha evidencia, se diseñen políticas de inclusión financiera más efectivas. En conclusión, en el Perú, la población con 
menores ingresos tiende a utilizar en igual proporción instrumentos financieros que otros grupos, a pesar de las grandes diferencias en ingresos. Esto es un potencial problema considerando que es la población más pobre aquella que cuenta con el menor nivel de educación financiera, sobre todo en lo referido a la aplicación de las tasas de interés que constituye la información más relevante para la toma de decisiones de endeudamiento cotidiano, a través del uso de la tarjeta de crédito. Por lo tanto, es imprescindible el desarrollo de medidas que coadyuven a fortalecer la educación financiera en el país, para lo cual es necesaria la generación de evidencia.

\section{REFERENCIAS}

Annabi, A., González-Ramírez, J. y Müller, F. (2018). What Determines Financial Knowledge among College Students? Journal of Financial Education, 44(2), 344-366.

Arango, L., y Cardona-Sosa, L. (2019). Tarjetas de crédito en personas de ingresos medios y bajos en Colombia: ¿qué determina su uso? (No. 1089). Banco de la Republica de Colombia.

Barboza, G., Smith, C. y Pesek, J. (2016). Assessing Financial Literacy, Gender Gap and Cognitive Differences. Journal of Financial Education, 42(3-4), 205-242.

Bertaut, C. y Haliassos, M. (2006). Credit cards: facts and theories. The economics of consumer credit, 181-237.

Caballero, G. (2018). Sobreendeudamiento y exoneración legal de los saldos insolutos en el procedimiento concursal del consumidor. Revista Ius et Praxis, 24(3), 133-172. http://www.revistaiepraxis.cl/index.php/iepraxis/article/view/ 1179/585

Ceballos-Mina, O., E., y Santiago-Ayala, L., E. (2019). Efectos de las tarjetas de crédito en la estructura de consumo y la desigualdad de los hogares mexicanos. Revista Finanzas y Política Económica, 11(2), 299-320. http:// dx.doi.org/10.14718/ revfinanzpolitecon.2019.11.2.5

Chan, R. Y. K. (1997). Demographic and attitudinal differences between active and inactive credit cardholdersthe case of Hong Kong. International Journal of Bank Marketing.

Encuesta Nacional de Demanda de Servicios Financieros y Nivel de Cultura Financiera en el Perú (2016). Recuperado de: https://www.sbs.gob.pe/Portals/0/jer/ ESTUDIOS-SOBRE-INCLUSI\%C3\%93N-FINANCIERA/Informe-de-Resultados.pdf
Instituto Nacional de Estadística e Informática. (2019). Encuesta Nacional de Hogares 2019. Recuperado de: https://www.inei.gob.pe/

Kim, H. y DeVaney, S. A. (2001). The determinants of outstanding balances among credit card revolvers. Financial Counseling and Planning, 12(1), 67-77.

Ponce, A., Seira, E., \& Zamarripa, G. (2017). Borrowing on the Wrong Credit Card? Evidence from Mexico. The American Economic Review, 107(4), 1335-1361.

Real Academia Española. (2020). Crédito. En Diccionario panhispánico del español jurídico. Recuperado de: https://dpej.rae.es/lema/cr\%C3\%A9dito2

Real Academia Española. (2020). Tarjeta de crédito. En Diccionario panhispánico del español jurídico. Recuperado de: https://dpej.rae.es/lema/tarjeta-de-cr\%C3\%A9dito

Sánchez, E. y Cisneros, A. (2020). Evolución del mercado de tarjetas de crédito. Revista Moneda del Banco Central de Reserva del Perú, 181, 23-27. https://www.bcrp. gob.pe/docs/Publicaciones/Revista-Moneda/moneda-181/moneda-181-04.pdf

Sarin, N. (2019). Making consumer finance work. Columbia Law Review, 119(6), 1519-1596. doi:10.2307/26794350

Seira, E., Elizondo, A. y Laguna-Müggenburg, E. (2017). Are Information Disclosures Effective? Evidence from the Credit Card Market. American Economic Journal: Economic Policy, 9(1), 277-307.

Superintendencia de Banca, Seguros y Administradoras Privadas de Fondos de Pensiones (2020). Reporte número de tarjetas de crédito por empresa bancaria. Recuperado de: https://www.sbs.gob.pe/app/stats_net/ stats/EstadisticaBoletin Estadistico.aspx? $\mathrm{p}=1 \#$

Zandi, G., Mansori, S. y Boon Hai, O. (2019). The Effect of Demographic Variables and Ownership on Credit Card Market in Malaysia. International Journal of Financial Research, 10(5), 359-369. Recuperado de: http://ijfr.sciedupress.com 